\title{
Demora no diagnóstico de câncer de mama de mulheres atendidas no Sistema Público de Saúde
}

\author{
Delay in breast cancer diagnosis on women assisted in \\ the Public Health System
}

\author{
Maria Cristina Traldi ${ }^{1}$, Priscila Galvão², Sirlei Siani de Morais ${ }^{3}$, \\ Márcia Regina Campos da Costa Fonseca ${ }^{1}$
}

\begin{abstract}
Resumo
Introdução: Apesar dos esforços, a mortalidade por câncer de mama se mantém elevada no Brasil e é mais frequente em regiões em que o acesso aos serviços de saúde é dificultado ou inexistente. Uma das recomendações do Instituto Nacional do Câncer (INCA) para reduzir a mortalidade é melhorar o acesso ao diagnóstico, realizando-o em até 60 dias após a suspeita. Objetivo: Estabelecer o tempo decorrido (em dias) entre a suspeita e o diagnóstico do câncer de mama, subdividindo os intervalos entre os eventos: consulta médica na atenção básica; mamografia ou ultrassonografia; consulta médica especializada; biópsia; consulta médica para conclusão do diagnóstico. Métodos: Estudo descritivo e seccional, realizado com 45 mulheres atendidas em um serviço público de saúde, com diagnóstico de câncer de mama efetivado em 2013. Resultados: A média da demora foi de 142,6 $\pm 10,1$ dias (12-451), com $60 \%$ dos diagnósticos efetivados entre 120 e 180 dias, e chance de efetivação do diagnóstico em até 60 dias para $8,9 \%$ da amostra. Conclusão: Há necessidade de implementação efetiva das linhas de cuidado na rede de atenção oncológica, com pactuação dos fluxos entre os serviços para agilizar o acesso à assistência integral às mulheres.
\end{abstract}

Palavras-chave: neoplasias da mama; saúde da mulher; política de saúde.

\begin{abstract}
Introduction: Despite efforts, mortality from breast cancer remains high in Brazil and is most common in regions where access to health services is difficult or inexistent. One of the INCA's (Instituto Nacional do Câncer) recommendations to reduce mortality is to improve the early diagnosis for up to 60 days after the suspicious. Objective: To establish the elapsed time (in days) between the suspect and the breast cancer diagnosis, subgrouping the timeframe in the events of: primary care medical appointment; mammography and/or ultrasonography; specialized medical appointment; biopsy and; medical appointment for the diagnosis conclusion. Methods: The descriptive and sectional study was accomplished with samples from 45 women attending the public health service, diagnosed with breast cancer in 2013. Results: The average elapsed time was of 142.6 days \pm 10.1 (12-451), with $60 \%$ of diagnosis performed within 120 and 180 days and effective diagnosed chance of up to 60 days for $8.9 \%$ of the cases. Conclusions: A effective implementation of care lines in the cancer care network is in need, with connection between services to speed women access to comprehensive assistance.
\end{abstract}

Keywords: breast cancer; women's health; health policy.

\footnotetext{
${ }^{1}$ Departamento de Enfermagem, Faculdade de Medicina de Jundiaí - Jundiaí (SP), Brasil.

${ }^{2}$ Curso de Enfermagem, Faculdade de Medicina de Jundiaí - Jundiaí (SP), Brasil.

${ }^{3}$ Departamento de Saúde Coletiva, Faculdade de Medicina de Jundiá - Jundiaí (SP), Brasil.

Trabalho realizado no Ambulatório de Saúde da Mulher de Jundiaí - Jundiaí (SP), Brasil.

Endereço para correspondência: Maria Cristina Traldi - Rua das Camélias, 81 - Chácara Primavera - CEP: 13087-488 - Campinas (SP), Brasil Email: mcristraldi@gmail.com
}

Fonte de financiamento: nenhuma.

Conflito de interesses: nada a declarar. 


\section{INTRODUÇÃO}

Estima-se que 11,1 milhões de novos casos de câncer tenham sido diagnosticados no mundo em 2004, provocando 7,4 milhões de mortes, com o câncer de mama sendo o segundo tipo mais frequente na população feminina ${ }^{1}$. No Brasil, dos 576 mil novos casos de câncer esperados para 2015, estimava-se que 75 mil seriam de câncer de mama².

Em situação diversa à dos países desenvolvidos, onde a mortalidade decresce, o aumento da incidência do câncer de mama no Brasil tem sido acompanhado do aumento da mortalidade, o que pode ser atribuído ao diagnóstico da doença em estádios avançados, devido à dificuldade de acesso aos serviços de saúde $e^{3-7}$. O diagnóstico precoce está associado a melhores prognósticos e chances de cura

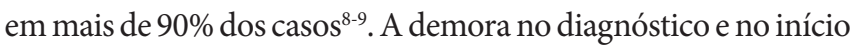
do tratamento tem sido associada à menor sobrevida - tempo superior a três meses entre o surgimento dos sintomas e o início do tratamento reduz a sobrevivência total em $12 \%^{4-6}$.

A demora no diagnóstico é definida como o tempo transcorrido entre o contato com o serviço de saúde na primeira consulta e a efetivação do diagnóstico, ao passo que a demora no tratamento é contabilizada entre a primeira consulta e o início do tratamento ${ }^{4,10,11}$.

Visando à redução da mortalidade e as repercussões físicas, psíquicas e sociais do câncer de mama feminino, o Ministério da Saúde do Brasil, por meio do Instituto Nacional do Câncer (INCA), estabeleceu, desde a década de 1990, políticas no intuito de estruturar uma rede assistencial para estimular a detecção precoce e o tratamento oportuno do câncer de mama. $\mathrm{O}$ atual protocolo nacional para a prevenção do câncer de mama recomenda o exame mamográfico bianual de rastreamento para mulheres com idade superior a 50 anos, antecipando a rotina de exames às mulheres de 35 anos, se pertencentes ao grupo de risco ${ }^{12}$.

A Política Nacional de Atenção Oncológica (PNAO), instituída em 2005 e atualizada em 2013, reconhece o câncer como problema de saúde pública e define em 60 dias o tempo de demora entre o diagnóstico e o início do tratamento; contudo, mantém indefinido o tempo máximo de espera para a efetivação do diagnóstico ${ }^{13}$.

Em 2011, documento publicado pelo INCA com sugestões de inclusão na PNAO recomenda estabelecer prazo máximo de 60 dias para que mulheres com nódulo palpável na mama e outras alterações suspeitas possam receber diagnóstico ${ }^{14}$.

O tempo de demora na efetivação do diagnóstico do câncer de mama é uma possibilidade como indicador para a análise da efetividade das políticas públicas no país, mas são escassos os estudos nacionais envolvendo a temática ${ }^{15}$.

O objetivo deste estudo foi calcular o tempo decorrido entre a primeira consulta, que gerou a solicitação de exame de imagem nos serviços de atenção básica, até a confirmação do diagnóstico de câncer de mama. Secundariamente, objetivou-se estabelecer o tempo parcial de demora de cada evento (exame de mamografia e/ou ultrassonografia; consulta médica com especialista; biópsia; consulta médica de fechamento do diagnóstico).

\section{MÉTODO}

Estudo seccional descritivo, realizado entre usuárias de um ambulatório público de saúde da mulher de um município com aproximadamente 400 mil habitantes, localizado no interior do Estado de São Paulo, Brasil.

No ano de 2013, 53 mulheres foram diagnosticadas com câncer de mama no ambulatório em que foi realizado o estudo. Destas, $45(84,9 \%)$ compuseram a amostra e 8 mulheres não aceitaram participar da pesquisa. As participantes responderam a um questionário semiestruturado com questões que abordavam: aspectos sociodemográficos; a respeito do atendimento que gerou a solicitação do exame de imagem das mamas na atenção básica; do percurso até a conclusão do diagnóstico de câncer de mama. As entrevistas ocorreram em um espaço reservado do ambulatório. As datas das consultas e dos exames, assim como dados do estadiamento clínico (EC) da doença, foram coletadas dos prontuários arquivados no ambulatório.

O tempo total de demora foi calculado pela soma dos dias transcorridos entre a realização dos eventos: a) a consulta médica na atenção básica e a realização da mamografia e/ou ultrassonografia; b) a mamografia e/ou ultrassonografia e a consulta médica no serviço especializado; c) a consulta médica especializada e a biópsia; d) entre a data da biópsia e a consulta médica de confirmação do diagnóstico de câncer.

O EC da doença seguiu a classificação internacional TNM, adotada pelo INCA, em que: T indica tumor primário; $\mathrm{N}$, presença de linfonodos regionais; e $\mathrm{M}$, metástase, sem especificação das subclasses A, B, C, com os diagnósticos agrupados em EC0, ECI, ECII, ECIII e EC IV ${ }^{16}$.

O estudo foi submetido e aprovado pelo Comitê de Ética sob o Protocolo n ${ }^{\circ} 393.009$, de 12 de setembro de 2013, e cumpriu rigorosamente as recomendações da Resolução no 466/12 e da Declaração de Helsinque. Todas as mulheres da amostra assinaram o Termo de Consentimento Livre e Esclarecido.

Para cálculo da demora no diagnóstico foi utilizado o método de sobrevida de Kaplan-Meier, que pode ser aplicado a quaisquer desfechos que sejam dicotômicos e que ocorram apenas uma vez durante o seguimento, oportunidade descrita como "análise de tempo até o evento" ${ }^{17}$. O valor-p foi calculado pelo teste de Wilcoxon.

\section{RESULTADOS}

As mulheres diagnosticadas com câncer de mama são, em sua maioria, brancas $(62,2 \%)$, com ensino fundamental $(66,7 \%)$, vivem com o(a) companheiro(a) (86,7\%) e possuem filhos (88,9\%), com expressivo percentual de aposentadas ou pensionistas (46,7\%). A média de idade das participantes foi de 59,3 $\pm 12,7$ anos (32-86 anos), com predominância da doença entre as mulheres na faixa dos 50 aos 69 anos (60\%) (Tabela 1).

A maioria expressiva $(95,6 \%)$ residia em bairros que possuíam Unidades Básicas de Saúde (UBS) ou Unidades de Saúde da Família (USF), e 88,9\% utilizavam regularmente o serviço de saúde para 
Tabela 1. Tempo de espera para o diagnóstico segundo as variáveis sociodemográficas, de estadiamento da doença e de utilização dos serviços de atenção básica de saúde em mulheres com câncer de mama, São Paulo-Brasil, 2013

\begin{tabular}{|c|c|c|c|c|c|}
\hline \multirow{2}{*}{ Características } & \multicolumn{5}{|c|}{ (Tempo total espera) } \\
\hline & $\mathbf{N}$ & $\%$ & Média & DP & Valor-p \\
\hline Idade & & & & & 0,9622 \\
\hline$<50$ anos & 10 & 22,2 & 131,6 & 11,2 & \\
\hline 50-69 anos & 27 & 60,0 & 149,0 & 16,3 & \\
\hline$\geq 70$ anos & 8 & 17,8 & 134,6 & 26,2 & \\
\hline Cor & & & & & 0,733 \\
\hline Branca & 28 & 62,2 & 143,5 & 15,5 & \\
\hline Parda & 16 & 35,6 & 141,6 & 15,4 & \\
\hline Negra & 1 & 2,2 & 132,0 & - & \\
\hline Ocupação & & & & & 0,1706 \\
\hline Do Lar & 13 & 28,9 & 128,3 & 19,6 & \\
\hline Aposentada/pensionista & 21 & 46,7 & 163,8 & 18,0 & \\
\hline Trabalhadora em atividade & 11 & 24,4 & 119,0 & 14,9 & \\
\hline Instrução & & & & & 0,5926 \\
\hline Analfabeta & 5 & 11,1 & 104,0 & 23,6 & \\
\hline Fundamental & 30 & 66,7 & 146,2 & 14,4 & \\
\hline Médio & 8 & 17,8 & 158,1 & 25,1 & \\
\hline Superior & 2 & 4,4 & 122,5 & 6,5 & \\
\hline Estado civil & & & & & 0,4381 \\
\hline Sem companheiro & 6 & 13,3 & 152,2 & 20,6 & \\
\hline Com companheiro & 39 & 86,7 & 141,1 & 12,3 & \\
\hline Tem filhos? & & & & & 0,4241 \\
\hline Não & 5 & 11,1 & 156,4 & 26,4 & \\
\hline Sim & 40 & 88,9 & 140,9 & 12,0 & \\
\hline Estadiamento & & & & & 0,0323 \\
\hline ECI e II & 30 & 66,7 & 137,8 & 11,5 & \\
\hline ECIII & 10 & 22,2 & 184,7 & 28,6 & \\
\hline ECIV & 5 & 11,1 & 87,2 & 28,0 & \\
\hline Serviço de saúde no bairro? & & & & & 0,9582 \\
\hline Sim & 43 & 95,6 & 143,5 & 11,5 & \\
\hline Não & 2 & 4,4 & 122,0 & 10,0 & \\
\hline Usuária do serviço saúde? & & & & & 0,3854 \\
\hline Sim & 40 & 88,9 & 142,0 & 12,3 & \\
\hline Não & 5 & 11,1 & 147,2 & 11,7 & \\
\hline Usa serviço para consulta com generalista? & & & & & 0,801 \\
\hline Sim & 27 & 60,0 & 145,1 & 16,7 & \\
\hline Não & 18 & 40,0 & 138,7 & 11,7 & \\
\hline Usa serviço para consulta com ginecologista? & & & & & 0,0431 \\
\hline Sim & 33 & 73,3 & 134,2 & 13,8 & \\
\hline Não & 12 & 26,7 & 165,7 & 15,0 & \\
\hline Consulta periódica com ginecologista? & & & & & 0,2772 \\
\hline Sim & 37 & 82,2 & 139,0 & 12,4 & \\
\hline Não & 8 & 17,8 & 159,0 & 23,6 & \\
\hline Exame periódico Papanicolaou? & & & & & 0,0913 \\
\hline Sim & 34 & 75,6 & 153,1 & 12,7 & \\
\hline Não & 11 & 24,4 & 110,1 & 19,8 & \\
\hline Mamografia 2 anos antes do diagnóstico & & & & & 0,2734 \\
\hline Sim & 26 & 57,8 & 151,3 & 13,6 & \\
\hline Não & 19 & 42,2 & 130,7 & 18,2 & \\
\hline
\end{tabular}

Método Kaplan-Meier; Teste de Wilcoxon; DP - desvio padrão 
suas demandas de saúde, incluindo consultas com ginecologista $(73,3 \%)$ ou com médico generalista $(60 \%)$. A rede de atenção básica do município era composta por igual número de UBS, que dispunham de ginecologista, e de USF, que contavam com médico generalista.

Quanto aos hábitos de autocuidado com a saúde, as entrevistadas revelaram que costumavam passar por consultas médicas ginecológicas periódicas $(82,2 \%)$ e que realizavam o exame Papanicolaou (75,6\%). Além disso, pouco mais da metade $(57,8 \%)$ havia realizado mamografia dois anos antes da suspeita de câncer de mama.

Das 45 mulheres integrantes da amostra, uma (2,2\%) teve diagnóstico efetivado em ECI; 29 (64,4\%), em ECII; 10 (22,2\%), em ECIII; e 5 (11,1\%), em ECIV. Para calcular a média de tempo decorrido até o diagnóstico, a única integrante com diagnóstico em ECI foi agregada às de classificação ECII (Tabela 1).

A associação da demora e do estádio da doença apresentou significância estatística ( $\mathrm{p}=0,0323)$, com menor média de demora nas pacientes com o diagnóstico em ECIV, de 87,2 \pm 28,0 dias, e com maior média de demora entre as pacientes com estadiamento ECIII, de 184,7 $\pm 28,6$ dias. Também apresentou significância usar o serviço de atenção básica para consulta com ginecologista ( $\mathrm{p}=0,0431)$, com média de demora menor $(134,2 \pm 13,8$ dias $)$ para quem a realiza, comparada à das que não possuíam essa prática, que foi de 165,7 $\pm 15,0$.

A média de demora entre o atendimento no serviço de saúde e a confirmação do diagnóstico foi de 142,6 \pm 10,1 dias (12-451), o que equivale a quatro meses de espera, aproximadamente. Vinte e sete mulheres da amostra (60\%) tiveram seus diagnósticos efetivados com tempo de espera entre 120 e 180 dias, com chance de o diagnóstico se concretizar em 60 dias para $8,9 \%$ da amostra (Tabela 2).

O tempo médio de espera entre os encaminhamentos foi superior a 30 dias, e o evento que mais contribuiu com a demora no diagnóstico foi a consulta médica no serviço especializado, com média de 46,3 dias (Tabela 3).

\section{DISCUSSÃO}

Pesquisas sobre a demora no diagnóstico e tratamento do câncer de mama no Brasil são ainda escassas e apresentam metodologias distintas tanto na construção da trajetória quanto nos testes estatísticos aplicados na análise, dificultando

Tabela 2. Demora (em dias) até a confirmação do diagnóstico de câncer de mama em usuárias do sistema público de saúde, São Paulo-Brasil, 2013

Demora do

diagnóstico (em dias)

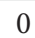

30

60

90

120

150

180

210

280

${ }^{*} \mathrm{DP}$ - desvio padrão

\section{Probabilidade de diagnóstico}

0,00

2,2

8,9

20,0

46,7

64,4

80,0

84,4

95,6
DP* Probabilidade de não diagnóstico

100

97,8

91,1

80,0

53,3

35,6

20,0

15,6

4,4

\section{N sem diagnóstico Diagnósticos}

Tabela 3. Distribuição das medidas de tendência central e de dispersão da demora, em dias, entre os intervalos desde a consulta na atenção básica até o diagnóstico de câncer de mama, São Paulo-Brasil, 2013

\begin{tabular}{ccccc}
\hline Medidas & \multicolumn{4}{c}{ Demora entre os atendimentos (em dias) } \\
\hline Medidas & $\begin{array}{c}\mathbf{C M}^{*} \text { na AB } \\
\mathbf{M M G}^{* * *} / \mathbf{U S G}^{* * * *}\end{array}$ & $\begin{array}{c}\text { Resultados exames } \\
\text { CM especialista }\end{array}$ & $\begin{array}{c}\text { CM especialista e biópsia } \\
\text { Biópsia e o diagnóstico }\end{array}$ \\
Média & 35,9 & 46,3 & 39,4 & 33 \\
DP & 37,6 & 54,7 & 44,3 & 32,6 \\
Mediana & 42,5 & 52,5 & 42 & 43,5 \\
Mínimo & 0 & 0 & 6 & 0 \\
Máximo & 147 & 319 & 241 & 208 \\
\hline
\end{tabular}

*Consulta Médica; ${ }^{* *}$ Atenção Básica; ${ }^{* * *}$ Mamogragia; ${ }^{* * *}$ Ultrassonografia 
a generalização dos resultados ${ }^{15}$. $\mathrm{O}$ acesso ao diagnóstico e ao tratamento varia de região para região do país, dependendo de variáveis geográficas e sociais relacionadas à distância entre a residência e os serviços, ao nível de informação das mulheres e à disponibilidade de serviços. O mapa da mortalidade por câncer de mama em mulheres mostra a influência desses fatores, indicando que ela é maior nas regiões em que a incidência da doença é elevada e os recursos médicos para diagnóstico precoce e tratamento adequado são de difícil acesso ou mesmo inexistentes ${ }^{18}$.

A média de demora de 142,6 dias para o diagnóstico do câncer de mama mostrou-se longa e possivelmente comprometedora de um prognóstico favorável à sobrevida das mulheres brasileiras, ou seja, 2,4 vezes o tempo recomendado pelo INCA, com chance de efetivação do diagnóstico em até 60 dias somente para $8,9 \%$ da amostra.

Pesquisa realizada com mulheres atendidas em um hospital público da capital paulista apontou demora no diagnóstico de cinco meses, aproximadamente 150 dias $^{19}$. Na América Latina, a capital colombiana apresentou mediana de demora de 91 dias $^{5}$, e na Malásia a demora no diagnóstico foi de cinco meses e meio ${ }^{20}$.

Em nosso estudo, o principal fator de demora no diagnóstico foi a espera para a consulta médica especializada no nível secundário de atenção à saúde, o que sugere fragilidade na efetivação da linha de cuidados oncológicos, definida como política pública e ainda em fase de implementação no país ${ }^{13,21}$.

A linha de cuidado pressupõe ações integradas dos recursos disponíveis: serviços, equipes e comunidade organizada, voltadas a atender às necessidades de saúde dos usuários, visando à redução da morbimortalidade e à melhora na qualidade de vida dos portadores da doença. Isso requer ações coordenadas que envolvam estratégias de prevenção, detecção precoce, tratamento oportuno e cuidados paliativos, complementadas por ações que perpassam os campos da educação e da comunicação em saúde, da vigilância do câncer e dos fatores de risco, além do desenvolvimento de pesquisa básica e aplicada ${ }^{22}$.

No que tange à assistência, as ações de rastreamento para o diagnóstico precoce são parte das atribuições dos serviços de atenção básica, ao passo que a investigação para a efetivação do diagnóstico e o tratamento fazem parte do conjunto de ações dos serviços especializados da atenção secundária e terciária, respectivamente. A concentração das ações de rastreamento na atenção básica ocorre não apenas por ser a principal porta de entrada para o sistema público de saúde, mas, fundamentalmente, em função da natureza da densidade tecnológica prevista para esse nível de atenção e da proximidade da equipe local com os usuários dos serviços, estreitada na medida do vínculo estabelecido no cotidiano da assistência ${ }^{13}$.

A linha de cuidado é a imagem pensada para expressar os fluxos assistenciais demandados em situações de saúde/doença para garantir o atendimento às necessidades de saúde. Aplicada ao câncer de mama, é o itinerário que a usuária percorre para garantir acesso à assistência integral de que necessita, incluindo nele as ações oferecidas nos três níveis de atenção e de assistência, além daquelas ofertadas pelas entidades comunitárias e sem fins lucrativos, integradas na rede para dar suporte social ao paciente e à família. Sua efetividade depende da pactuação do fluxo entre os serviços e da reorganização dos processos de trabalho ${ }^{21-22}$.

A quebra no fluxo dos serviços ou a inexistência de articulação entre eles costuma dificultar o percurso dos usuários na rede e atrasar a concretização das ações, retardando o diagnóstico, o início do tratamento ou até mesmo as ações de natureza social, fundamentais em situações de vulnerabilidade social.

O tempo gasto pelas mulheres em cada etapa da assistência expõe gargalos importantes na agenda de consulta de especialidade na atenção secundária e para a biópsia.

A média de 35,9 dias para a realização da mamografia e/ou ultrassonografia foi o terceiro motivo da demora no percurso até o diagnóstico. Contudo, mostrou-se menor que o tempo médio verificado anteriormente no mesmo município, de 60 dias $^{23}$. Foi inferior também ao de $62 \%$ dos 12 países da América Latina, incluindo o Brasil, que participaram de recente estudo envolvendo serviços públicos e privados, em que a demora foi inferior a três meses ${ }^{24}$.

A mamografia continua sendo o melhor instrumento para rastreamento do câncer de mama, além de ser fundamental a garantia do acesso facilitado para reduzir os índices de diagnósticos tardios ${ }^{21}$.

Nos países da União Europeia, o parâmetro do tempo de espera para a mamografia é o do guia da European Breast Cancer Network (EBCN), que estabelece prazo de 15 dias úteis entre a realização e a emissão do laudo para a mamografia de rastreamento, e de cinco dias úteis para as sintomáticas. Além de definir prazos para as mamografias, o European Guideline define indicadores de demora entre cada evento nas etapas diagnósticas e terapêuticas do câncer de mama ${ }^{25}$.

Na América Latina, mais de $90 \%$ dos países não dispõem de qualquer política ou diretriz nacional sobre direito das mulheres à triagem do câncer de mama por meio da mamografia ${ }^{24}$.

Além da demora na mamografia, os resultados revelam que pouco mais da metade $(57,8 \%$, ou $26 / 45)$ das mulheres possuíam mamografia realizada dois anos antes do diagnóstico de câncer de mama, sugerindo falhas no programa de rastreamento desse tipo de câncer no âmbito da atenção básica. Estima-se que apenas 9,6\% da população feminina brasileira com 50 anos ou mais de idade tenha realizado mamografia de rastreamento em 2010. Mesmo diante do subregistro no sistema de informação, a taxa está muito aquém dos 70\% recomendados pela Organização Mundial da Saúde ${ }^{26}$. 
A associação significativa $(\mathrm{p}=0,0431)$ entre a demora no diagnóstico e o hábito de utilizar o serviço de atenção básica para realizar consultas ginecológicas sugere maior acesso ao diagnóstico (31,5 dias de diferença entre as médias), quando comparado às que não realizam consulta ginecológica.

Embora sem associação estatística significativa, destaca-se que a demora no diagnóstico (média $=145,1$ dias) foi maior entre as mulheres que realizam consulta médica com generalista, em comparação com as que se consultam com ginecologistas na atenção básica (média $=138,7$ dias). Esse achado merece ser aprofundado em novas pesquisas, uma vez que o fluxo de encaminhamento não difere, em teoria, entre um tipo de serviço e outro (UBS e USF) da atenção básica até a especializada.

Também mostrou associação estatística significante $(\mathrm{p}=0,0323)$ ter diagnóstico em ECI/ECII e menor demora no diagnóstico, corroborando achado de estudo realizado em hospital público da capital paulista ${ }^{19}$. Os resultados reforçam a importância da atenção básica no rastreamento do câncer de mama e possivelmente do vínculo entre as usuárias e os serviços, o que contribui favoravelmente na construção da linha de cuidados dentro da rede de atenção oncológica ${ }^{21-22}$.

Por ser uma amostra pequena e proveniente dos diagnósticos de um único centro e em um único ano, a generalização dos resultados deste estudo deve ser feita com cautela.

\section{CONCLUSÃO}

A demora média de 142,5 dias - 2,4 vezes maior que o tempo máximo recomendado de 60 dias para o diagnóstico do câncer de mama - reforça a necessidade de implementação efetiva das linhas de cuidado na rede de atenção oncológica, com pactuação dos fluxos entre os serviços para agilizar o acesso das mulheres à assistência integral, conforme preconizam as políticas públicas.

\section{REFERÊNCIAS}

1. World Health Organization. The global burden of disease: 2004 update. Geneve: WHO Press; 2008

2. Brasil. Ministério da Saúde. Estimativa 2014: incidência do Câncer no Brasil [Internet]. Rio de Janeiro: INCA; 2013 [citado em 2013 abr 5]. Disponível em: http://www.inca.gov.br

3. Brasil. Ministério da Saúde. Secretaria de Atenção à Saúde. Instituto Nacional do Câncer. Controle do câncer de mama: documento de consenso [Internet]. Rio de Janeiro: INCA; 2013 [citado em 2013 fev 3]. 39 p. Disponível em: http://www2.inca.gov.br/wps/wcm/connect/tiposdecancer/site/home/ mama/literatura.

4. Ayala ALM. Sobrevida de mulheres com câncer de mama, de uma cidade no sul do Brasil. Rev Bras Enferm. 2012;65(4):566-70. http://dx.doi. org/10.1590/S0034-71672012000400003. PMid:23258674.

5. Piñeros MSPM, Sánchez MER, Fernando Perry MC, García MCOA, Ocampo MCR. Demoras em el diagnóstico y tratamiento de mujeres com câncer de mama em Bogotá, Colombia. Salud Publica Mex. 2011;53(6):47885.

6. Burgess CC, Ramirez AJ, Richards MA, Love SB. Who and what influences delayed presentation in breast cancer? Br J Cancer. 1998;77(8):1343-8. http://dx.doi.org/10.1038/bjc.1998.224. PMid:9579844.

7. Ramirez AJ, Westcombe AM, Burgess CC, Sutton S, Littlejohns P, Richards MA. Factors predicting delayed presentation of symptomatic breast cancer: a systematic review. Lancet. 1999;353(9159):1127-31. http://dx.doi. org/10.1016/S0140-6736(99)02142-X. PMid:10209975.

8. ESHRE Capri Workshop Group. Hormones and breast cancer. Hum Reprod Update. 2004;10(4):281-93. http://dx.doi.org/10.1093/humupd/dmh025. PMid:15192054.

9. Medeiros C, Mendes K, Scavone M. Percepções sobre o câncer de mama [Internet]. Instituto Avon; 2012 [citado em 2013 abr 17]. Disponível em: http://www.institutoavon.org.br/wpcontent/themes/institutoavon/pdf/ pesquisa_instituto_avon_2012.pdf.

10. Barber MD, Jack W, Dixon JM. Diagnostic delay in breast cancer. Br J Surg. 2004;91(1):49-53. http://dx.doi.org/10.1002/bjs.4436. PMid:14716793.

11. Dang-Tan T, Franco EL. Diagnosis delays in childhood cáncer: a review. Cancer. 2007;110(4):703-13. http://dx.doi.org/10.1002/cncr.22849. PMid:17620277.

12. Brasil. Ministério da Saúde. Instituto Nacional do Câncer. Diretrizes para a detecção precoce do câncer de mama no Brasil [Internet]. Rio de Janeiro: INCA; 2015 [citado em: 2015 ago 13]. Disponível em: http://www2.inca.gov. $\mathrm{br} / \mathrm{wps} / \mathrm{wcm} / \mathrm{connect} /$ agencianoticias/site/home/noticias/2015/ministerio_ saude_aprova_diretrizes_nacionais_deteccao_precoce_cancer_mama

13. Brasil. Portaria $n^{\circ} 874$, de 16 de maio de 2013. Institui a Política Nacional para a Prevenção e Controle do Câncer na Rede de Atenção à Saúde das Pessoas com Doenças Crônicas no âmbito do Sistema Único de Saúde (SUS). Diário Oficial da União, Brasília, 16 de maio de 2013.

14. Brasil. Ministério da Saúde. Instituto Nacional do Câncer. Recomendações do INCA para reduzir a mortalidade por câncer de mama no Brasil [Internet]. Brasília: MS; 2014 [citado em 2014 jun 7]. Disponível em: www1.inca.gov. br/inca/Arquivos/recomendacoes_ca_mama_2013.pdf.

15. Barros AF, Uemura G, Macedo JLS. Atraso no diagnóstico e tratamento do câncer de mama e estratégias para a sua redução. Femina. 2012;40(1):31-6b.

16. Brasil. Ministério da Saúde. Instituto Nacional do Câncer. ABC do Câncer: abordagens básicas para o controle do câncer. Rio de Janeiro: INCA; 2011 [citado em 2014 jun 7]. Disponível em: http://bvsms.saude.gov.br/bvs/ controle_cancer

17. Flecher RH, Flecher SW. Epidemiologia clínica, elementos essenciais. 4. ed. Porto Alegre: Artmed; 2006.

18. Oliveira EXG, Melo ECP, Pinheiro RS, Noronha CP, Carvalho MS. Acesso à assistência oncológica: mapeamento dos fluxos origem-destino das internvenções e dos atendimentos ambulatoriais: o caso do câncer de mama. Cad Saude Publica. 2011;27(2):317-26. http://dx.doi.org/10.1590/ S0102-311X2011000200013. PMid:21359468.

19. Trufelli DC, Miranda VC, Santos MBB, Fraile NMP, Pecoroni PG, Gonzaga SFR, et al. Análise do atraso no diagnóstico e tratamento do câncer de mama em um hospital público. ABM Rev Assoc Med Bras. 2008;54(1):72-6. http://dx.doi.org/10.1590/S0104-42302008000100024 
20. Norsa'adah B, Rampal KG, Mohd A. Diagnosis delay of breast cancer and its associated factors in Malaysian women. BMC Cancer. 2011;11(141):240711.

21. Brasil. Ministério da Saúde. Instituto Nacional do Câncer. Diretrizes para a detecção precoce do câncer de mama no Brasil. Rio de Janeiro: INCA; 2015 [citado em 2015 jul 28]. Disponível em: http://www2.inca.gov.br/wps/ wcm/connect/acoes_programas/site/home/nobrasil/programa_controle_ cancer_mama/textos-referencia

22. Malta DC, Merhy EE. O percurso da linha do cuidado sob a perspectiva das doenças crônicas não transmissíveis. Interface Comunicacao Saude Educ. 2010;14(34):593-605. http://dx.doi.org/10.1590/S1414-32832010005000010.

23. Maia EMC, Maia LB, Valente FM, Machado RB, Borges JBR. Tempo decorrido entre a primeira consulta e o tratamento definitivo nos casos de câncer de mama no Sistema de Saúde Público da cidade de Jundiaí. Rev Bras Mastol. 2006;1:23-6.
24. Gazap E, Buzaid AC, De La Garza J, Schwartsmann D, Vallejos C, Guercovich A. Breast câncer in Latin America, results of the Latin America and Caribean Society os Medical Oncology/Breast Cancer Research Foudation Expert Survey. Cancer. 2008;113(8 Supl):2359-65. PMid:18837031.

25. European Union. European guidelines for quality assurance in breast cancer screening and diagnosis. 4. ed. Luxembourg; 2006 [citado em 2013 jul 12]. Disponível em: http://europa.eu.int/comm/dgs/health_consumer/ index_en.htm

26. Lee BL, Liedke PER, Barrios CH, Simon SD, Finkelstein DM, Goss PE. Breast cancer in Brazil, present status and future goals. Lancet Oncol. 2012;13(3):e95-102. http://dx.doi.org/10.1016/S1470-2045(11)70323-0. PMid:22381937.
Recebido em: Mar. 08, 2016 Aprovado em: Jun. 14, 2016 